\title{
FREQUENCY-DOMAIN ANALYSIS OF RESONANT CURRENT CONTROLLERS FOR ACTIVE POWER FILTERS
}

\author{
L. R. Limongi ${ }^{1}$, D. Roiu ${ }^{2}$, R. Bojoi ${ }^{3}$, A. Tenconi ${ }^{3}$ \\ ${ }^{1}$ Universidade Federal de Pernambuco, Department of Electrical Engineering, Recife-PE - Brazil \\ ${ }^{2}$ FIAT Research Centre, Torino-TO - Italy \\ ${ }^{3}$ Politecnico di Torino, Department of Electrical Engineering, Torino-TO - Italy \\ Emails: leonardo.limongi@ufpe.br,radu.bojoi@polito.it
}

\begin{abstract}
This paper performs a frequency-domain analysis of three different resonant current controllers for active power filters. Respect to previous papers, the novelty consists of a stability analysis of the closed loop system using the Nyquist criterion. To perform this analysis, the time delay introduced by the inverter and the sampling due to the digital control are taken into account in the current controller closed loop transfer function. This procedure demonstrates the importance and effectiveness of the delay compensation to maintain the whole system stability. Experimental results are presented for a $25 \mathrm{kVA}$ shunt active power filter prototype to confirm the theoretical analysis.
\end{abstract}

\section{Keywords - Active Power Filters, Current control.}

\section{INTRODUCTION}

In the last decades, harmonic filtering has become an important research topic as a consequence of the intensive use of the non-linear electrical loads that degrade the power quality in the distribution grids. Passive filters are a cheap and simple solution to reduce the harmonic content injected into the grid. However, the passive filters (also known as harmonic trap filters) have some drawbacks that sometimes limit their usage, such as resonance problems and poor transient performance [1].

The Active Power Filter (APF) emerged as an efficient solution for harmonic current compensation, featuring capabilities of compensating load imbalance and reactive power [1], [2]. The APF principle has been known by three decades but its feasibility and application in industry were limited by the development of fast switching devices [3]. Once this barrier was overcome, the research related to APFs was focused on many issues regarding either new topologies [3], [4]- [6] or control issues, such as the current reference generation based on power theories [3], [7], [8].

In recent years, a special attention has been paid to current controllers for APFs [9]- [24]. A performance comparison of different digital current controllers has been presented in [13], where the main criterions for performance evaluation were the Total Harmonic Distortion (THD) of the mains line current and the transient performance for fast load variations. It has been shown that the dead-beat [11] and the PI controller in synchronous reference frame [12], fail in obtaining zero steady-state error. On the other side, the frequency selective

Artigo submetido em 30/04/2010. Revisado em 12/11/2010. Aceito para publicação em 16/11/2010 por recomendação do editor João Onofre P. Pinto. current controllers exhibit better performance and can be implemented with off-the-shelf DSP controllers [13].

The literature reports different frequency selective controllers [14]- [24]. These methods can be applied when the harmonic spectrum of the load current to be compensated consists of harmonics having well-known orders and sequences. For three-phase diode or thyristor front-end rectifiers, these harmonics are of order $h=\mp 6 n+1$ (for $n=1,2, \ldots)$ of the fundamental frequency, as shown in Table I. The signs "+" and "-" indicate the direct and inverse sequences, respectively.

The most promising frequency selective controllers are those based on resonant regulators [15]- [18], [22]- [24]. The first frequency selective controller employed the so called Sinusoidal Signal Integrator (SSI) introduced in [15]. The SSI is a resonant element that obtains zero steady-state error for sinusoidal input having a frequency equal to the SSI resonance frequency. In [18], multiple SSIs have been used to individually compensate for the harmonics of order $h=\mp 6 n+1$ (for $n=1,2, \ldots$ ) in stationary frame. In [22], the SSIs have been implemented in Synchronous Reference Frame (SRF). This approach halves the numbers of the required SSIs since one SSI is able to compensate for simultaneously the positive and negative sequences of the SSI resonant frequency. The harmonics $h=\mp 6 n+1$ (for $n=1,2, \ldots$ ) in stationary frame become $h=\mp 6 n$ (for $n=1,2, \ldots$ ) in synchronous frame (Table I) and consequently can be compensated with one SSI tuned on the $6 n$ (for $n=1,2, \ldots$ ) harmonic. The latest approach concerning the use of SSIs is found in [23], [24], where the SSIs are also implemented in SRF but along with a decoupling scheme.

The goal of this paper is to present a frequency-domain analysis of resonant current controllers for shunt APFs. The current control schemes considered in this analysis are the following ones:

- Proportional and Sinusoidal Signal Integrators in Synchronous Reference Frame (P-SSI-SRF) [22];

- Stationary frame controller with Proportional regulator and Sinusoidal Signal Integrators (P-SSI) [18];

- PI controller with Resonant regulators (PI-RES) in Synchronous Reference Frame [24];

Compared with previous works found in literature [13], [23][25] it will be clearly shown how the transfer functions of each current control have been obtained. In addition, an analysis based on closed-loop frequency response will show the system behavior for each current control, addressing some intrinsic characteristics in terms of magnitude and phase for 
TABLE I

Current Harmonics for Three-Phase Rectifiers

\begin{tabular}{c|c|c}
\hline \hline $\begin{array}{c}\text { Harmonic in }(\alpha, \beta) \\
\text { stationary reference } \\
\text { frame }\end{array}$ & Sequence & $\begin{array}{c}\text { Harmonic order in (d,q) } \\
\text { synchronous reference frame } \\
\text { rotating at fundamental frequency }\end{array}$ \\
\hline+1 (fundamental) & positive & DC \\
\hline-5 & negative & -6 \\
\hline+7 & positive & +6 \\
\hline$\ldots$ & $\ldots$ & $\ldots$ \\
\hline$-6 \mathrm{n}+1$ & negative & $-6 \mathrm{n}$ \\
\hline $6 \mathrm{n}+1$ & positive & $+6 \mathrm{n}$ \\
\hline
\end{tabular}

high-order frequencies. This approach is quite different from those used in previous works [23], [25] due to the fact that the computation time delay is taken into account for the analysis. This leads to quite different results. In fact, the computation time delay must be taken into account due to the fact that this is the cause of the system instability as will be shown in this paper. Finally, the limits in terms of stability for each current control will be determined using the Nyquist stability criterion for the first time for these class of controllers. All the theoretical analysis will be confirmed by experimental results provided for a $25 \mathrm{kVA}$ APF prototype compensating up to the $49^{\text {th }}$ harmonic of a non-linear load.

\section{FREQUENCY RESPONSE ANALYSIS}

The frequency selective current controllers analyzed in this paper were originally implemented in different referential frames: the P-SSI controller was implemented in stationary frame while the P-SSI-SRF and the PI-RES were implemented in the fundamental or $d q$ frame. However, all the controllers should be analyzed in the same referential frame to have a fair comparison.

The harmonic frame representation is useful to understand the principle of the current regulators. In fact, the use of multiple rotating frames (reference frames rotating with a angular speed of $(\mp 6 n+1) w_{e}$ for $n=1,2, \ldots$, where $\omega_{e}$ is the fundamental frequency) makes possible the transformation of each harmonic to be compensated in a dc signal. Therefore, the harmonic compensation can be performed using a simple Proportional-Integral (PI) controller for each harmonic of interest.

Although the harmonic reference is convenient to explain the current controllers principle, the frequency response analysis should be performed in another referential frame. For this reason, all current controllers analyzed in this paper will be first represented in harmonic frame and then transformed in the fundamental referential frame where the frequencydomain analysis will be performed.

\section{A. P-SSI-SRF Controller}

A generic PI controller that regulates the current of an RL load in harmonic frame is shown in Figure 1. The converter is considered here as an unity gain. The transfer function of this current controller is:

$$
H_{P I}^{h}(s)=k_{p h}+\frac{k_{i h}}{s}
$$

where $h=\mp 6 n+1$ (for $n=1,2 \ldots$ ) is the harmonic reference frame needed to transform each harmonic of interest in a

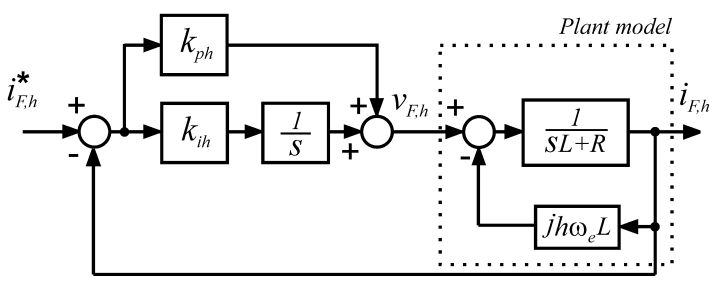

Fig. 1. Proportional-Integral (PI) current control in harmonic reference frame $h$.
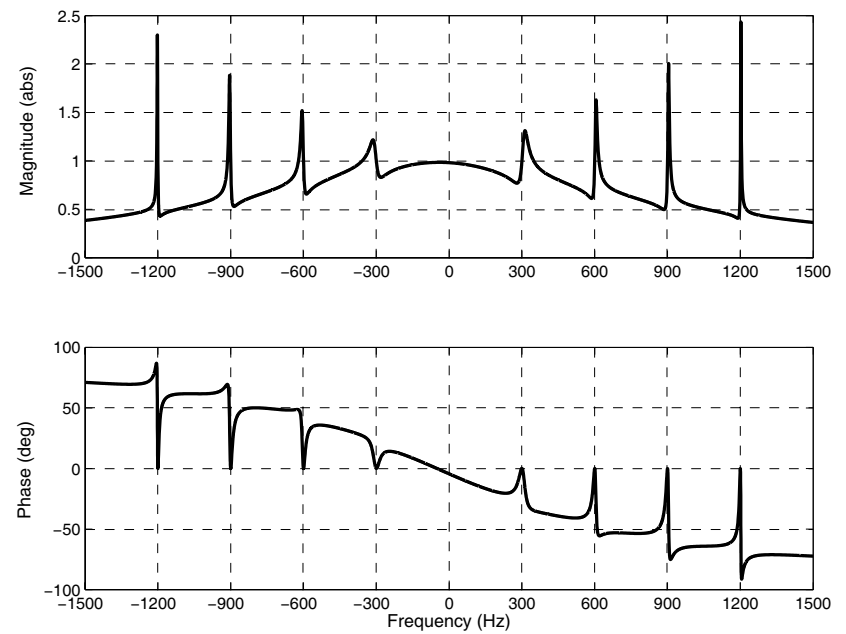

Fig. 2. Frequency response of P-SSI-SRF control loop in fundamental frame. Parameters: $k_{p h}=0.2, k_{i h}=k_{p h} R / L, L=350 \mu \mathrm{H}$, $R=22 m \Omega$.

dc quantity. The signs "+" and "-" indicate the direct and inverse sequences, respectively. In this situation, the PI control shown in (1) is able to achieve zero steady-state error. Since a rotational transformation in time domain is equivalent with a frequency shift in frequency domain, (1) can be transformed from the harmonic frame to fundamental frame using a frequency shift of $-j w_{e}(h-1$ ), where $h=\mp 6 n+1$ (for $n=1,2 \ldots)$ :

$$
H_{P I_{\mp}}^{1}(s)=\frac{k_{p h} s+k_{i h}+j k_{p h}( \pm 6 n) w_{e}}{s+j( \pm 6 n) w_{e}} n=1,2 \ldots
$$

The superposition of a positive sequence PI controller $H_{P I_{+}}^{1}$ and a negative sequence PI controller $H_{P I_{-}}^{1}$ results in the transfer function of P-SSI-SRF controller in fundamental frame:

$$
H_{S S I}^{1}(s)=2 k_{p h}+\frac{2 k_{i h} s}{s^{2}+\left(6 n w_{e}\right)^{2}} \quad n=1,2 \ldots
$$

where the superscript " 1 " indicates the fundamental reference frame. The RL plant transfer function in fundamental frame is:

$$
G_{\text {plant }}(s)=\frac{1}{L s+R+j w_{e} L}
$$

The closed-loop transfer function using the P-SSI-SRF current control has been determined from (3) and (4) for $n=1,2,3,4$ and the frequency response is shown in Figure 2. The parameters used in this frequency response analysis are 
derived from the experimental setup shown in a later section. The magnitude gains and phases at the frequencies of interest (i.e. at the resonant frequencies) have the desired values, 1 and 0 respectively, but in the vicinity of these points, there are magnitude peaks higher than unity with phase variations. However, this characteristic does not affect the system stability, as demonstrated in the next sections.

\section{B. P-SSI Controller}

The P-SSI controller compensates for the harmonics of order $h=\mp 6 n+1$ (for $n=1,2 \ldots$ ) (as the P-SSI-SRF controller does), but is also able to compensate for the inverse sequences of these harmonics (with order $h=-(\mp 6 n+1)$, for $n=1,2 \ldots)$. This feature can be useful to compensate for unbalanced loads. So, two set of harmonics are considered for this analysis: $h_{1}=\mp 6 n+1$ (for $n=1,2 \ldots$ ) and its inverse sequence $h_{2}=-(\mp 6 n+1)$ (for $\left.n=1,2 \ldots\right)$.

We consider the same PI controller in harmonic frame of Figure 1 with $h=h_{1}=\mp 6 n+1$ (for $n=1,2 \ldots$ ). By performing a frequency shift of $-j w_{e}(h-1)$ in $(1)$ to transform from harmonic frame to fundamental frame, it results:

$$
H_{P I_{1}}^{1}(s)=\frac{k_{p h} s+k_{i h}+j k_{p h}( \pm 6 n) w_{e}}{s+j( \pm 6 n) w_{e}} n=1,2 \ldots
$$

Note that (2) and (5) are identical. Now, considering the PI controller in harmonic frame with $h=h_{2}=-(\mp 6 n+1)$ (for $n=1,2 \ldots)$ and performing a frequency shift of $-j w_{e}(h-1)$ in (1), it results:

$$
H_{P I_{2}}^{1}(s)=\frac{k_{p h} s+k_{i h}+j k_{p h}(\mp 6 n+2) w_{e}}{s+j(\mp 6 n+2) w_{e}} n=1,2 \ldots
$$

The superposition of $H_{P I_{1}}^{1}$ and $H_{P I_{2}}^{1}$ yields the transfer function of a P-SSI controller referred to the fundamental $(d q)$ frame:

$$
H_{S S I}^{1}(s)=H_{P I_{1}}^{1}(s)+H_{P I_{2}}^{1}(s)
$$

The closed-loop transfer function using the P-SSI current control has been determined from (4) and (7) for $n=1,2,3,4$ and the closed-loop frequency response is shown in Figure 3. It can be seen that the frequency responses of P-SSI and PSSI-SRF are almost identical at the frequencies of interest.

As can be noted from Figure 3, the frequency response presents more resonant peaks compared with the P-SSI-SRF since the P-SSI is able to compensate for both sequences of a specified harmonic, i.e. $(\mp 5, \mp 7, \ldots, \mp 6 n+1)$. For this reason, the P-SSI controller is able to compensate for an imbalanced non-linear load [18]. In addition, transforming (7) to the stationary frame yields:

$$
H_{S S I}^{0}(s)=2 k_{p h}+\frac{2 k_{i h} s}{s^{2}+\left(h w_{e}\right)^{2}}
$$

where $h=(\mp 6 n+1)$ for $(n=1,2 \ldots)$ and the superscript " 0 " means stationary frame. This expression is exactly the current regulator proposed in [18].

\section{PI-RES Controller}

The PI-RES controller is also derived from a PI controller in harmonic frame but considering a decoupling scheme, as
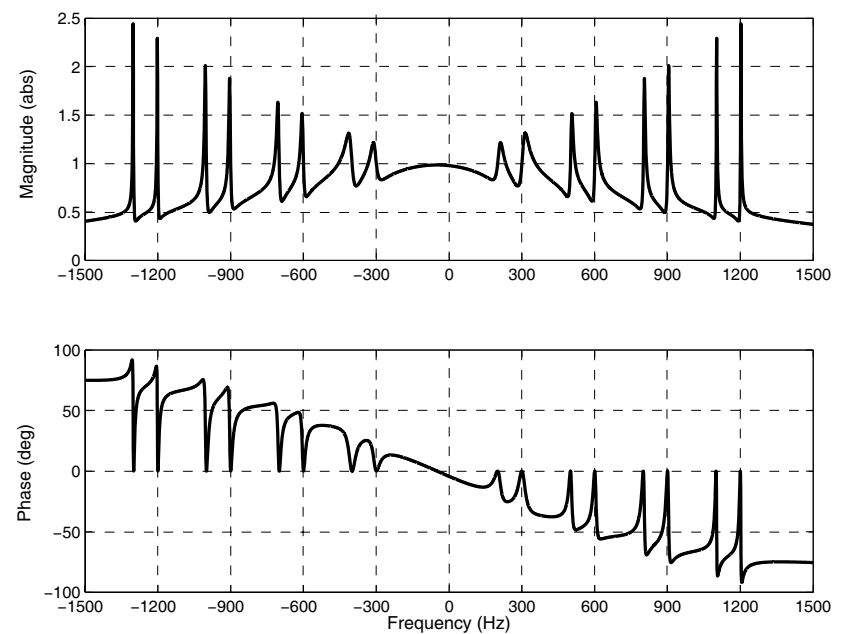

Fig. 3. Frequency response of P-SSI in fundamental frame. Parameters: $k_{p h}=0.2, k_{i h}=k_{p h} R / L, L=350 \mu \mathrm{H}, R=22 m \Omega$.

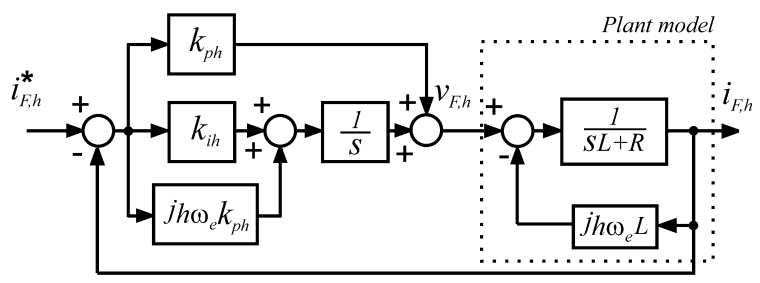

Fig. 4. PI current control with a decoupling scheme in harmonic frame $h$.

shown in Figure 4 [26]. Following this scheme, the controller transfer function is:

$$
H_{P I}^{h}=k_{p h}+\frac{k_{i h}+j h w_{e} k_{p h}}{s}
$$

where $h=\mp 6 n+1$ (for $n=1,2 \ldots$ ). If (9) is transformed from harmonic frame to fundamental frame using a frequency shift of $-j w_{e}(h-1)$, it results:

$$
H_{P I_{\mp}}^{1}=\frac{k_{p h} s+k_{i h} \pm j k_{p h} w_{e}}{s \pm j(6 n) w_{e}} n=1,2 \ldots
$$

The superposition of a positive PI controller $H_{P I_{+}}^{1}$ and a negative sequence PI controller $H_{P I_{-}}^{1}$, determines the following transfer function in the fundamental frame:

$$
H_{S S I}^{1}=\frac{2 k_{p h} s^{2}+2 k_{i h} s+2 j k_{p h} w_{e} s}{s^{2}+\left(6 n w_{e}\right)^{2}} n=1,2 \ldots
$$

It can be noted that this transfer function presents a coupling term between the $d$ and $q$ axes. However, the PI-RES controller transfer function proposed in [24] is slightly different from (11) since it does not contain the " $j$ " term:

$$
H_{S S I}^{1}=\frac{2 k_{p h} s^{2}+2 k_{i h} s}{s^{2}+\left(6 n w_{e}\right)^{2}} n=1,2 \ldots
$$

In fact, it is well known that the plant in fundamental frame has a coupling term equal to $j w_{e} L$. So, in order to eliminate this 


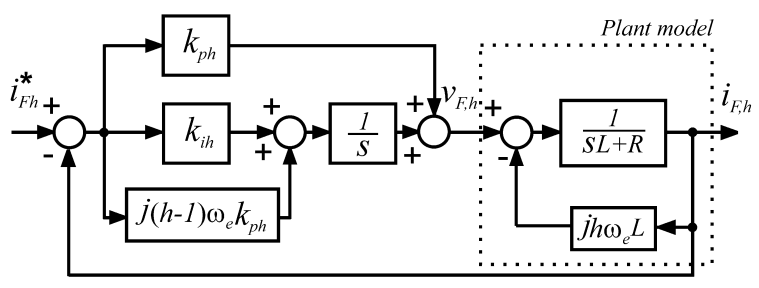

Fig. 5. PI current control with a modified decoupling scheme in harmonic frame $h$.

coupling term, the scheme of Figure 4 must be used (exactly as done in [26]) and the transfer function of the current control must be (11). For this reason, (12) does not obtain full decoupling in fundamental frame since it corresponds to the scheme of Figure 5. To prove that, we consider the transfer function of a PI current controller as shown in Figure 5:

$$
H_{P I}^{h}(s)=k_{p h}+\frac{k_{i h}+j(h-1) w_{e} k_{p h}}{s}
$$

where $h=\mp 6 n+1$ (for $n=1,2 \ldots$ ). If (13) is transformed from harmonic frame to fundamental frame using a frequency shift of $-j w_{e}(h-1)$, it results:

$$
H_{P I_{\mp}}^{1}=\frac{k_{p h} s+k_{i h}}{s \pm j(6 n) w_{e}} n=1,2 \ldots
$$

The PI-RES transfer function in fundamental frame $H_{S S I}^{1}$ is the superposition of $H_{P I_{+}}^{1}$ and $H_{P I_{-}}^{1}$ as follows:

$$
H_{S S I}^{1}=\frac{2 k_{p h} s^{2}+2 k_{i h} s}{s^{2}+\left(6 n w_{e}\right)^{2}} n=1,2 \ldots
$$
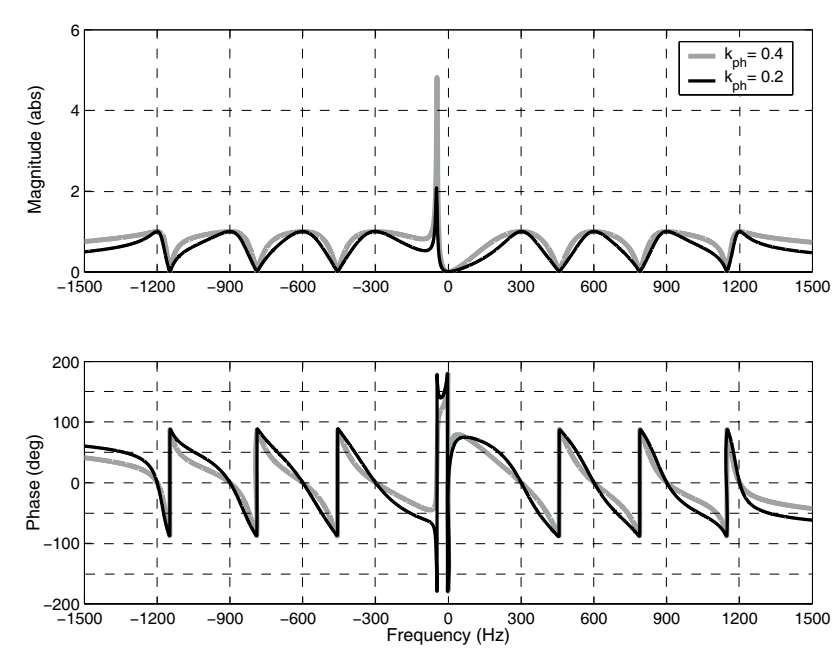

Fig. 6. Frequency response of PI-RES control loop in fundamental frame using (15). Parameters: $k_{i h}=k_{p h} R / L, L=350 \mu H, R=22 m \Omega$, $k_{p h}=0.4$ and 0.2 .

The closed-loop transfer function using the PI-RES current control has been determined from (4) and (15) for $n=1,2,3,4$. The closed-loop frequency response is shown in Figure 6 for two values of $k_{p h}$. It can be seen in both cases that the
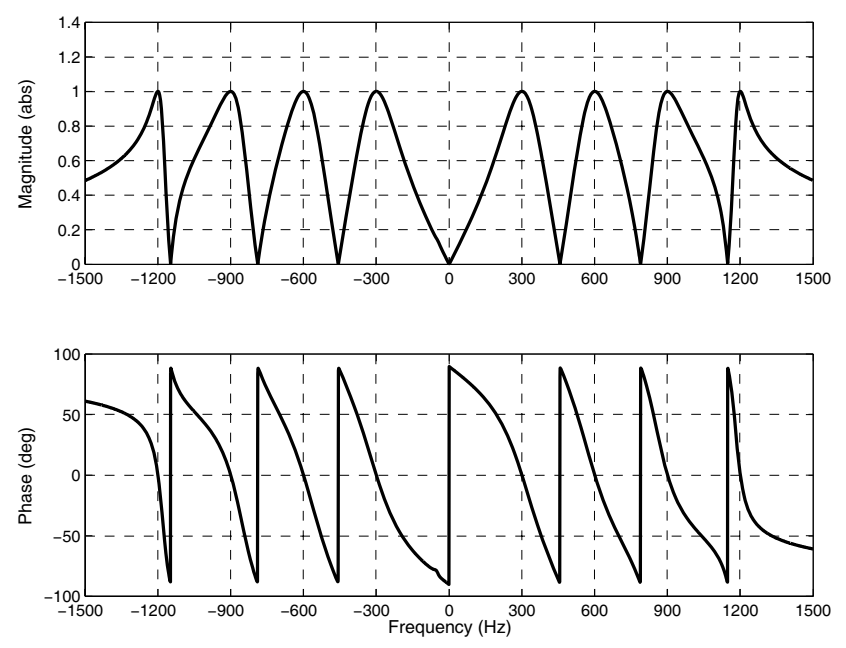

Fig. 7. Frequency response of PI-RES control loop in fundamental frame using (11). Parameters: $k_{p h}=0.2, k_{i h}=k_{p h} R / L, L=350 \mu H$, $R=22 m \Omega$.

magnitude gains and phases at the frequencies of interest (i.e. at the resonant frequencies) have the desired values, 1 and 0 respectively. There are no peaks magnitudes higher than unity and no significant phases variations in the vicinity of the harmonic frequencies of interest ( $h=\mp 6 n$, for $n=1,2 \ldots$ ). However, there is an anomalous magnitude resonant peak at $-50 \mathrm{~Hz}$ (which depends on the $k_{p}$ value) for the $d$-axis and another one at $+50 \mathrm{~Hz}$ for the $q$-axis (not shown here). There is also an anomalous phase variation caused by the term in " $j$ " neglected in (15). This causes an asymmetry in the closedloop frequency response of the PI-RES current controller.

The resonant peaks at $\mp 50 \mathrm{~Hz}$ may have a negative influence if the current sampling is not perfect and introduces some DC offset. In this case, a second order harmonic is injected by the current control. That will be seen in fundamental frame as a $50 \mathrm{~Hz}$ component, which is exactly the frequency of anomalous peak magnitude for $q$-axis. On the other hand, if the current controller is implemented with (11), the frequency response becomes symmetrical and there is no anomalous peak as shown in Figure 7. Another option should the implementation of the closed-loop of Figure 4 in stationary frame. In this case, the transfer function of current controller becomes $\frac{2 k_{p h} s^{2}+2 k_{i h} s}{s^{2}+\left(h w_{e}\right)^{2}}$ with $h=\mp 6 n+1(n=1,2 \ldots)$, and the frequency response is also symmetrical, but doing this, the advantage of use one SSI controller to compensate for simultaneously two harmonics is lost.

\section{Frequency Response Analysis Considering a Time Delay}

For discrete systems where the sampling frequency is equal with the switching frequency, a mean delay of $\tau=1.5 T_{s}$ (where $T_{s}$ is the sampling time) is introduced in the current control loop [27]. For this reason, the computation time delay is taken into account for a frequency response analysis as shown in Figure 8. The current controller transfer function $H_{S S I}^{1}$ contains one of equations (3), (7) and (15) (all of them in fundamental frame). The closed-loop frequency response for the P-SSI-SRF controller is shown in Figure 9. The time delay introduced in the control loop causes an abrupt phase 


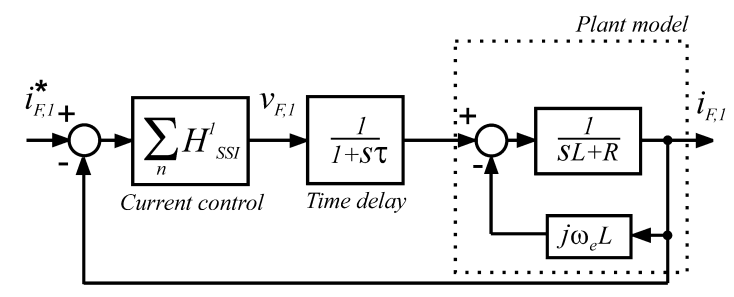

Fig. 8. Representation of a general current control scheme with a time delay.
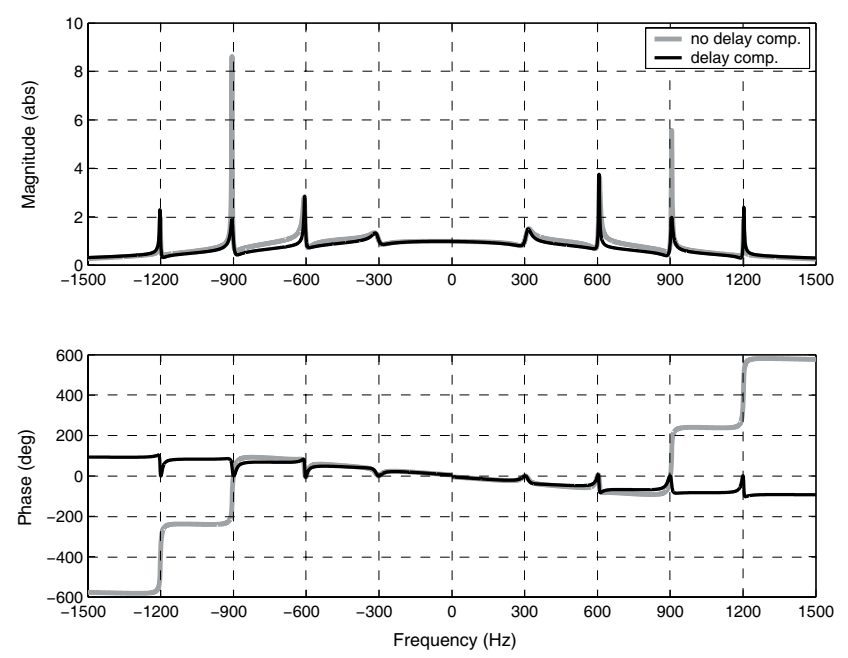

Fig. 9. Frequency response of P-SSI-SRF control loop in fundamental frame.

shift for high order harmonic frequencies (starting from the $17^{\text {th }}$ harmonic) which can lead to system instability if the compensation of these harmonics is required. In addition, the magnitude peak at the $17^{\text {th }}$ harmonic increases three times in relation to the same peak shown in Figure 2. On the other hand, when the time delay compensation is added to the control loop, the frequency response (also shown in Figure 9) becomes approximately equal to the case shown in Figure 2. The implementation of the time delay compensation is described in detail in [13], [22].

The same analysis has been performed for the P-SSI controller. This controller has presented a similar frequency response behavior when time delay is introduced, as shown in Figure 10. The increased phase-shift and magnitude peaks are also presented in the frequency response analysis. Therefore, the P-SSI and P-SSI-SRF present the same frequency response characteristics at the frequencies of interest $h=\mp 6 n$ $(n=1,2 \ldots)$.

For the PI-RES controller, the time delay produces an increasing of the magnitude peaks, as shown in Figure 11. In addition, an abrupt phase shift can be seen starting at the $29^{t h}$ harmonic that can lead the system to instability if the compensation of this harmonic is required. When the delay compensation is introduced in the control loop, the frequency response becomes approximately equal to the case shown in Figure 6.

\section{NYQUIST STABILITY ANALYSIS}

In former section, the frequency response analysis has shown some possible instability limits for the current controls due to the high phase shift for high order harmonics when the time delay is considered in the closed-loop model.

In this section, a stability criterion is used to investigate the behavior of the closed-loop transfer function of the scheme of Figure 8 in terms of absolute stability. The Nyquist criterion has been chosen due to the fact that it is more appropriate than root-locus for high-order systems [28]. Thus, the stability of the closed-loop current controls of Figure 8 is investigated by examining encirclements of the point $(-1, j 0)$ by the openloop polar plots [28].

The first analysis attempts to identify the stability limit of the current controls without delay compensation. The Nyquist plots for the P-SSI-SRF current control considering the first 2 harmonic pairs $\left(5^{t h}, 7^{\text {th }}\right.$ and $\left.11^{\text {th }}, 13^{\text {th }}\right)$ without delay compensation is shown in Figure 12. It is possible to see that the point $(-1, j 0)$ is not enclosed by the polar plot resulting in a stable system. Going further, introducing the third harmonic pair $\left(17^{\text {th }}, 19^{\text {th }}\right)$, the system becomes unstable because there is an encirclement of $(-1, j 0)$ as shown in Figure 13. On the other hand, when the delay compensation is used (Figure 14), the point $(-1, j 0)$ is not enclosed by the polar plot and the system is stable.

The Nyquist plots for the P-SSI control have shown the same stability limits of the P-SSI-SRF control (as suggested by the frequency response analysis) and for this reason, they are not shown here.

The Nyquist diagrams for the PI-RES control without delay compensation is shown in Figure 15 for the first four harmonic pairs $\left(\left(5^{t h}, 7^{t h}\right),\left(11^{t h}, 13^{t h}\right),\left(17^{t h}, 19^{t h}\right)\right.$ and $\left(23^{r d}\right.$, $\left.25^{\text {th }}\right)$ ). The system is stable because the point $(-1, j 0)$ is not enclosed by the polar plots. However, going beyond this limit, the system becomes unstable as shown in the Nyquist plots of Figure 16. The control loop becomes stable for these harmonics pairs only if the delay compensation is used, as shown in Figure 17. All the results obtained using the Nyquist criterion are in agreement with the frequency response analysis performed in the previous section, which means that the harmonic compensation limit without delay compensation for the P-SSI and P-SSI-SRF control is the $13^{t h}$ harmonic frequency. This limit is increased to the $25^{\text {th }}$ harmonic when PI-RES control is used. When the delay compensation is implemented, all current controls are stable and their performance are very similar [13].

\section{EXPERIMENTAL RESULTS}

A 25-kVA shunt APF prototype has been built, using an Insulated Gate Bipolar Transistor (IGBT) three-phase inverter with a switching frequency of $10 \mathrm{kHz}$. The current controllers considered in this paper have been included in a digital control scheme for an APF, with $10 \mathrm{kHz}$ of sampling frequency. The APF compensates a $55 \mathrm{~kW}$ non-linear load consisting of a diode front-end rectifier having an inductive load, as shown in Figure 18. The DC-link reference voltage of the IGBT inverter has been set at $730 \mathrm{~V}$. The inverter interface inductance, $L_{F}$, and the input load inductance, $L_{L}$, are equal to $250 \mu \mathrm{H}$. 

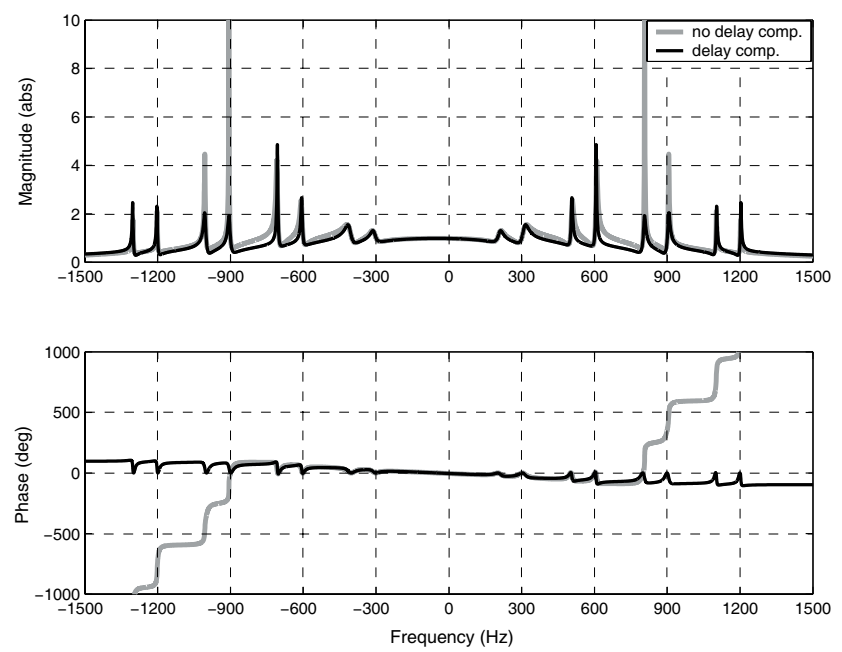

Fig. 10. Frequency response of P-SSI control loop in fundamental frame.
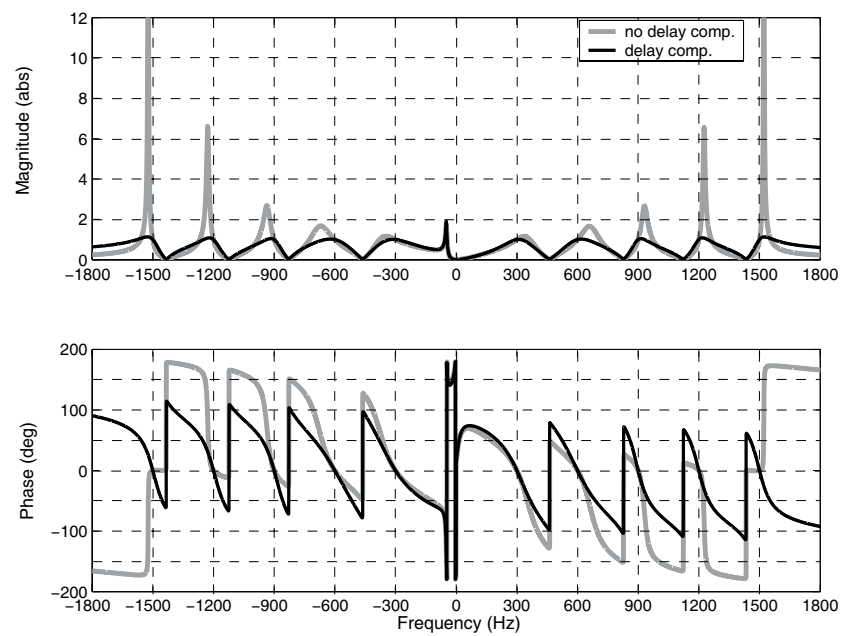

Fig. 11. Frequency response of PI-RES control loop in fundamental frame.
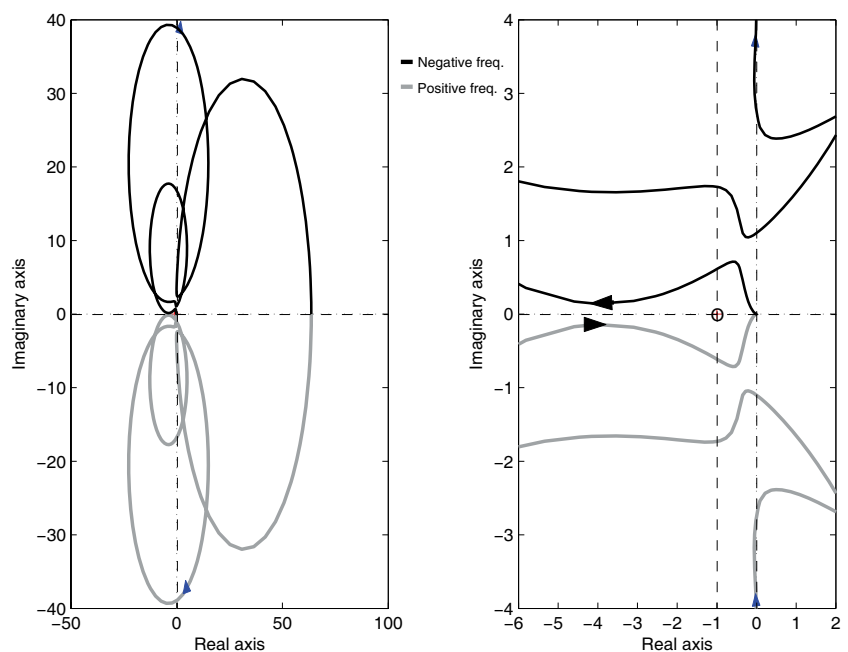

Fig. 12. From left to right: Nyquist diagram for P-SSI-SRF control without delay compensation considering the first 2 harmonic pairs and its zoom view around the critical point $(-1, \mathrm{j} 0)$. The control is stable.
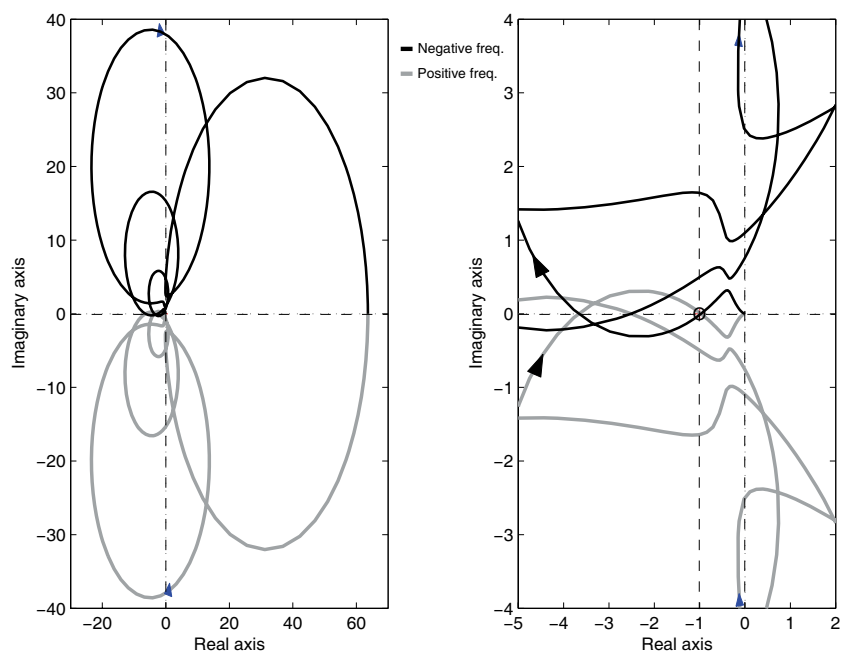

Fig. 13. From left to right: Nyquist diagram for P-SSI-SRF control without delay compensation considering the first 3 harmonic pairs and its zoom view around the critical point $(-1, \mathrm{j} 0)$. The control is unstable.
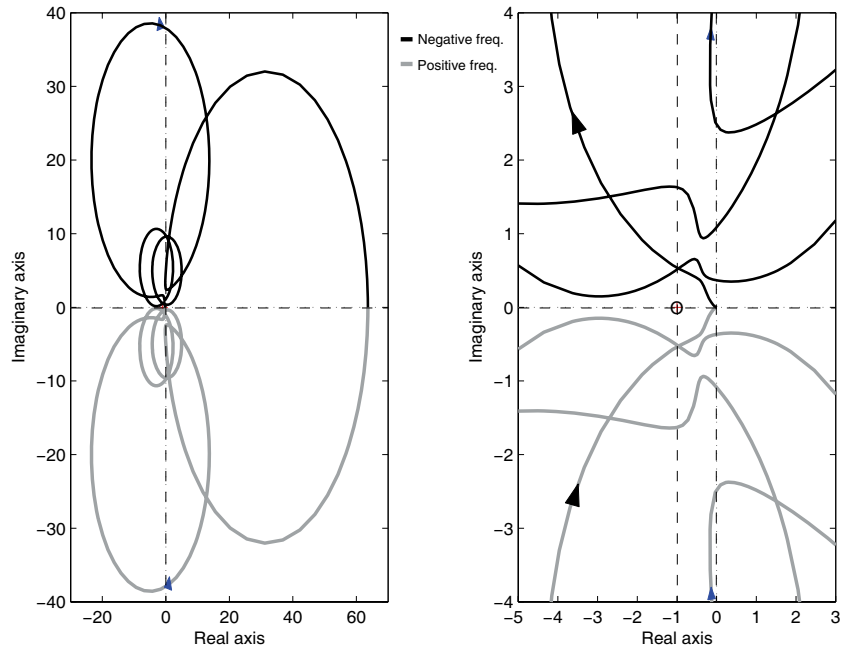

Fig. 14. From left to right: Nyquist diagram for P-SSI-SRF control with delay compensation considering the first 3 harmonic pairs and its zoom view around the critical point $(-1, \mathrm{j} 0)$. The control is stable.

The mains inductance is about $100 \mu \mathrm{H}$. The control system has been implemented using dSPACE DS1103 development board. Besides the current control block, the APF control scheme of Figure 18 consists of a reference generator, a Phase Locked Loop (PLL) scheme to detect the position of the PCC voltage vector and an outer voltage loop to regulate the DC link voltage.

It has been demonstrated in the previous sections that the frequency-domain analysis for P-SSI and P-SSI-SRF present the same behavior at frequencies of interest. In addition, the equivalency of both controllers in terms of steady-state and transient performance has also been reported in [13]. Therefore, this section will be concentrated in the analysis of the P-SSI-SRF and the PI-RES controllers.

The first experiment compares the performance of current controllers in terms of maximum harmonics to be mitigated 

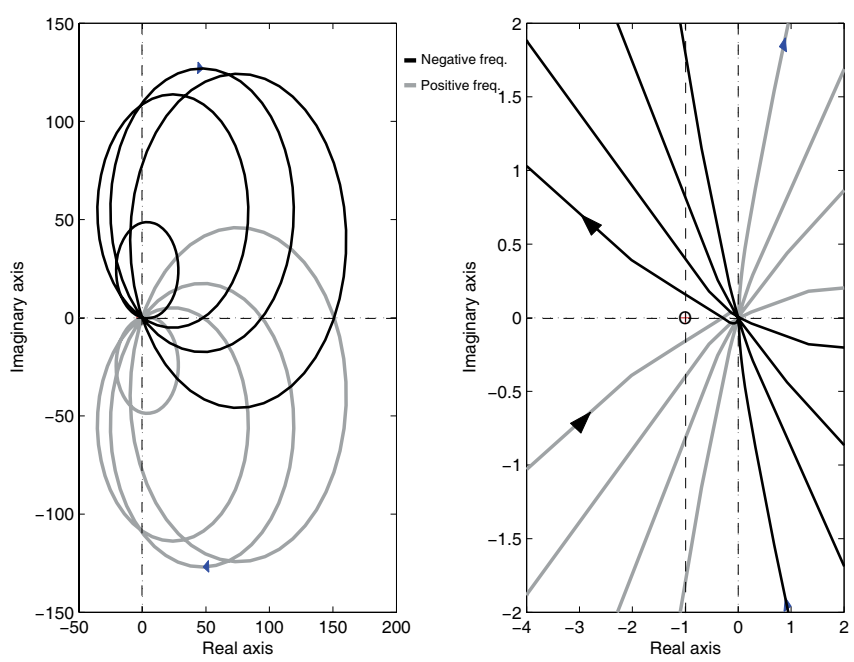

Fig. 15. From left to right: Nyquist diagram for PI-RES control without delay compensation considering the first 4 harmonic pairs and its zoom view around the critical point $(-1, j 0)$. The control is stable.
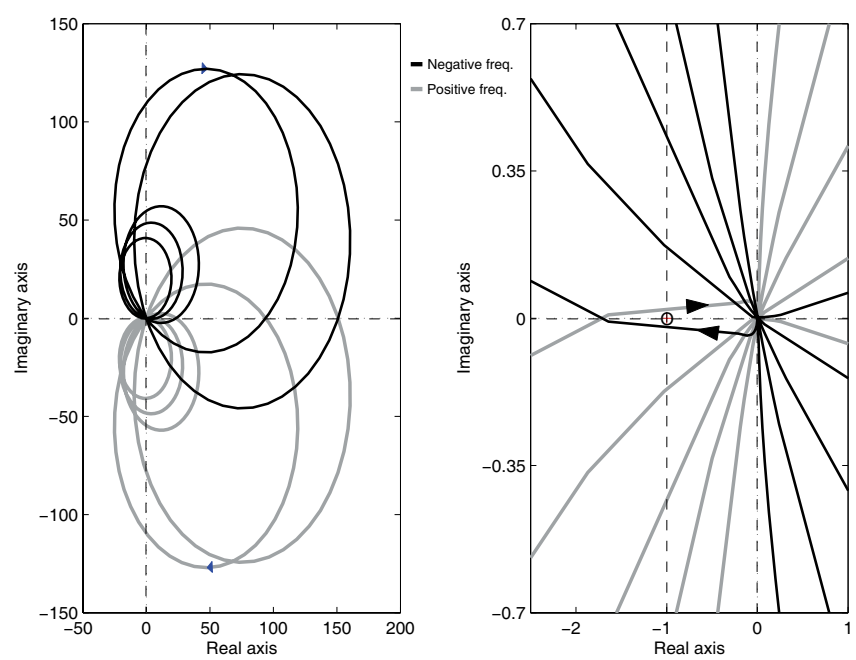

Fig. 16. From left to right: Nyquist diagram for PI-RES control without delay compensation considering the first 5 harmonic pairs and its zoom view around the critical point $(-1, \mathrm{j} 0)$. The control is unstable.

without delay compensation. The inductive load current waveform and its harmonic spectrum are shown in Figure 19. For the P-SSI-SRF controller, the first four harmonics, $h=\mp 6 n+1$ (for $n=1,2$ ) are completely compensated without any stability problems. However, as soon as the $17^{\text {th }}$ and $19^{\text {th }}$ harmonics are programmed to be compensated, their amplitudes start increasing and the system becomes unstable (Figure 20). As consequence, APF prototype goes into protection mode due to overcurrent. This is in complete agreement with the frequency response of Figure 9 (which shows a high phase shift at these frequencies) and the Nyquist plot of Figure 13. The same test has been performed for the PI-RES controller and demonstrated that is possible to compensate up to the $25^{\text {th }}$ harmonic without any delay compensation as shown by the frequency response of Figure

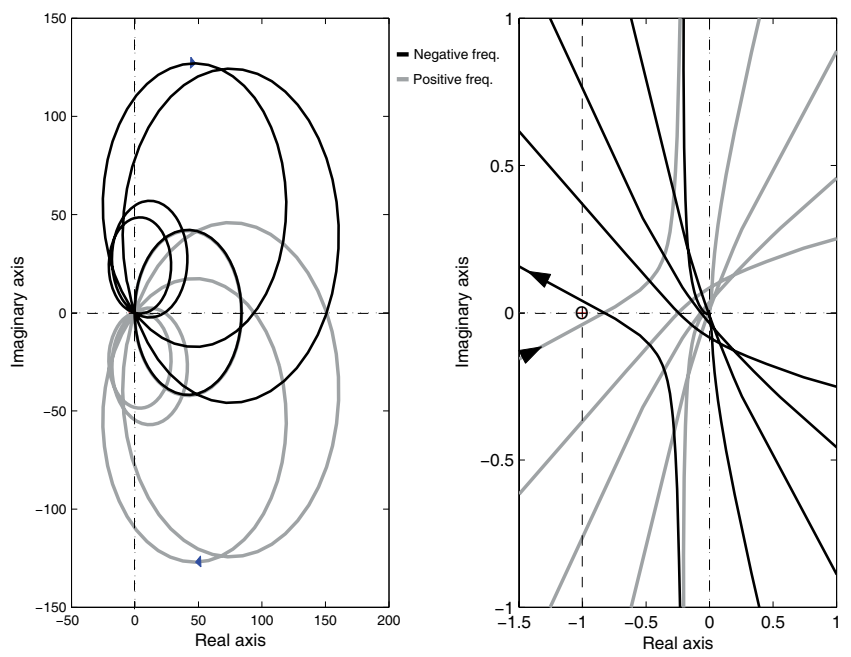

Fig. 17. From left to right: Nyquist diagram for PI-RES control with delay compensation considering the first 5 harmonic pairs and its zoom view around the critical point $(-1, j 0)$. The control is stable.

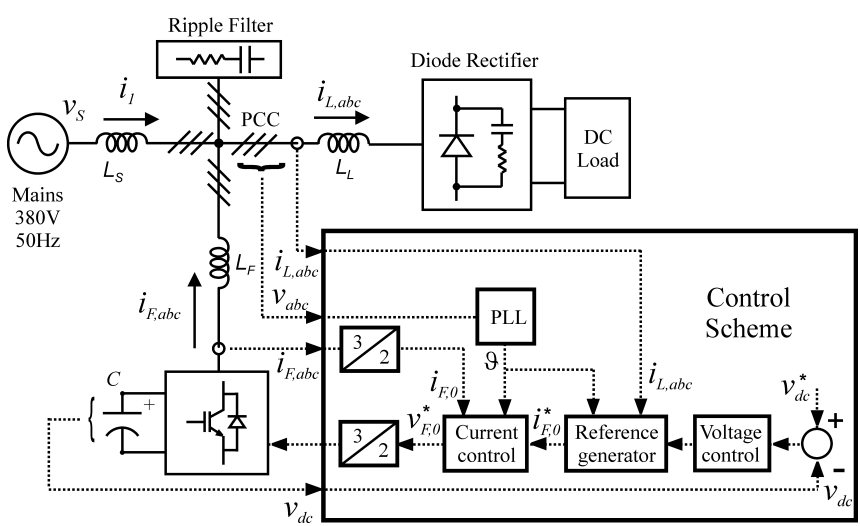

Fig. 18. Active Power Filter general scheme: Power plant and control scheme.

11 and the Nyquist plot of Figure 15. However, when the $29^{t h}$ and $31^{\text {st }}$ harmonics are programmed to be compensated, their amplitudes start increasing and the system becomes unstable (Figure 21), tripping the APF overcurrent protection. This experiment confirms the result found in the Nyquist plot of Figure 16.

When the delay compensation is used in the P-SSI-SRF and PI-RES controllers, the control-loop becomes stable and able to compensate for the desired harmonics confirming the results of Nyquist plots of Figure 14 and 17. There is no significant performance difference between the current controllers if the delay compensation is implemented correctly. For example, considering the compensation up to $25^{t h}$ harmonic, the P-SSISRF controller needs delay compensation for the $17^{\text {th }}, 19^{\text {th }}$, $23^{r d}$ and $25^{\text {th }}$ harmonics while PI-RES controller is stable. In this case, the THD of the mains current for the P-SSISRF and PI-RES controllers is $2.57 \%$ and $2.59 \%$ respectively. The steady-state waveforms for the P-SSI-SRF and PI-RES controllers are shown in Figures 22 and 23. Considering the compensation up to $37^{\text {th }}$ harmonic, the P-SSI-SRF controller needs delay compensation starting from the $17^{\text {th }}$ harmonic 
TABLE II

P-SSI-SRF control

\begin{tabular}{c|c|c}
\hline \hline Compensated harmonics & up to $25^{\text {th }}$ & up to $37^{\text {th }}$ \\
\hline THD & $2.57 \%$ & $2.27 \%$ \\
\hline Delay compensation & starting at $17^{\text {th }}$ & starting at $17^{\text {th }}$ \\
\hline \hline
\end{tabular}

TABLE III

PI-RES control

\begin{tabular}{c|c|c}
\hline \hline Compensated harmonics & up to $25^{t h}$ & up to $37^{t h}$ \\
\hline THD & $2.59 \%$ & $2.17 \%$ \\
\hline Delay compensation & not used & starting at $29^{t h}$ \\
\hline \hline
\end{tabular}

up to the $37^{\text {th }}$, while the PI-RES controller needs a delay compensation starting at $29^{\text {th }}$ harmonic up to the $37^{\text {th }}$. In this scenario, the P-SSI-SRF and PI-RES controllers present a THD of $2.27 \%$ and $2.17 \%$ respectively. Both tests are summarized in Tables II and III. It can be concluded that when the delay compensation is used, the steady state performance of P-SSI-SRF and PI-RES are almost identical.

Regarding the harmonic compensation limit, both controllers were able to compensate up to the $49^{\text {th }}$ harmonic. Beyond this frequency, the digital control fails due to the small number of samples in a period (less than four samples for the sampling frequency of $10 \mathrm{kHz}$ ). The Fourier analysis for these cases are shown in Figures 24, 25 and 26 with another scale.

It must be emphasized here that in a real implementation with an industrial DSP controller, the maximum number of harmonics that can be compensated is limited more or less at the $25^{\text {th }}$ harmonic for both P-SSI-SRF and PI-RES, while the P-SSI controller will have less compensation capability due to the higher number of required SSI regulators. However, the P-SSI is able to compensate for imbalanced non-linear loads [18], while the P-SSI-SRF can deal with imbalanced loads only for the fundamental frequency [22]. On the contrary, the PI-RES cannot deal with imbalanced loads even for the fundamental frequency since it uses a PI controller in the fundamental frame.

Another important issue regarding this family of current controllers comes from the fact that all of them are sensitive to frequency variations. However, the major consequences of this problem are mainly presented in the controlling of harmonic currents of higher order as for example the $24^{\text {th }}$ harmonics (in fundamental frame). In this case, a variation of $0.25 \mathrm{~Hz}$ in the fundamental frequency means a deviation of $6 \mathrm{~Hz}$ for the $24^{\text {th }}$ harmonic frequency. So, the SSI tuned in the $24^{\text {th }}$ harmonic will deal with this frequency variation. In principle, it is possible to tune the SSI frequencies using a simple PLL scheme. Another alternative is to implement the current controllers in harmonic frame where there is no need to determine the resonant frequencies. In this case the controllers are represented by simple PI's controllers with multiple rotational transformations whose angles are computed by a PLL.

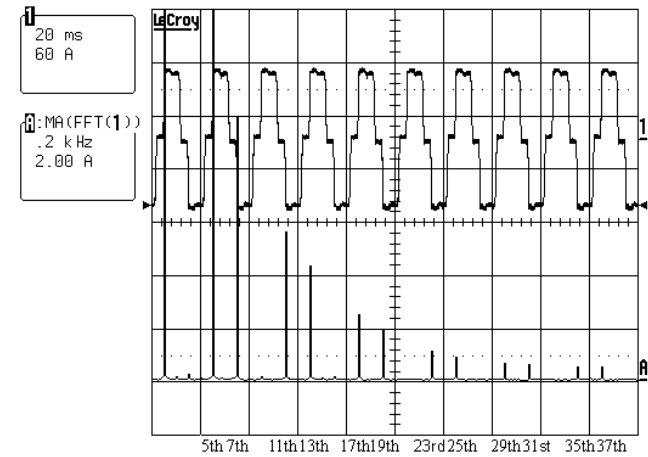

Fig. 19. Inductive load current waveform (phase A) and its Fourier analysis.

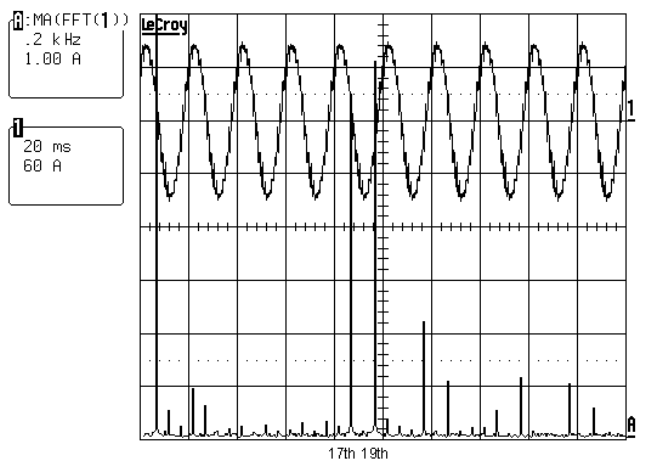

Fig. 20. Fourier analysis of mains current for P-SSI-SRF control with no delay compensation.

\section{CONCLUSIONS}

This paper performs a frequency-domain analysis of three different resonant current controllers for active power filters. These controllers can also be used for other grid-connected converters, such as active rectifiers and inverters for distributed power generation.

With respect to previous papers, the novelty consists of a stability analysis of the closed loop system using the Nyquist criterion. To perform this analysis, the time delay introduced by the inverter and the digital sampling were taken into

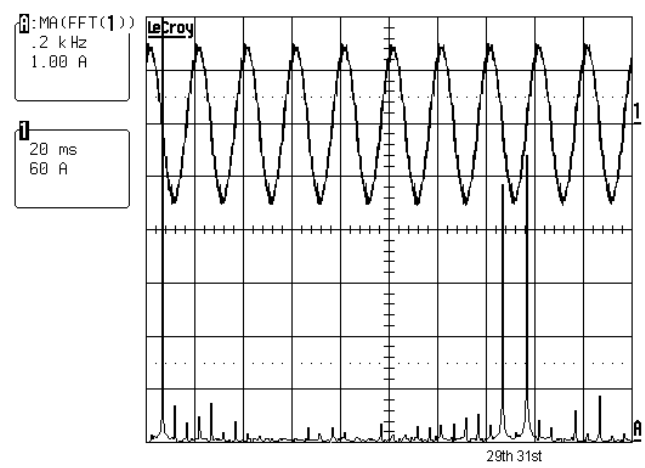

Fig. 21. Fourier analysis of mains current for PI-RES control with no delay compensation. 


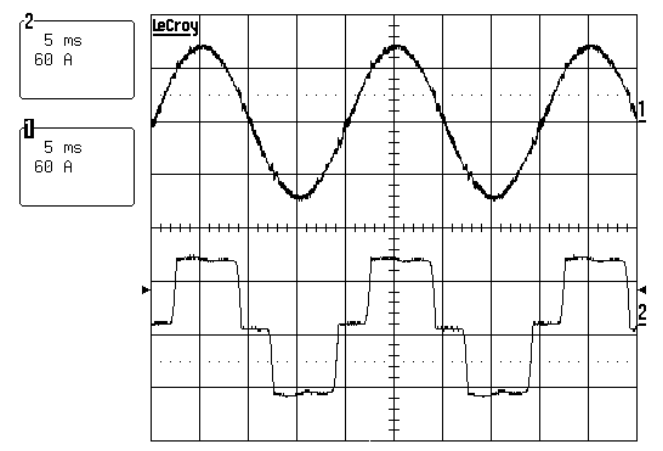

Fig. 22. Steady-state operation compensating up to $25^{\text {th }}$ harmonic for P-SSI-SRF controller. Trace 1: $i_{S a}(\mathrm{~A})$. Trace 2: $i_{L a}$ (A).

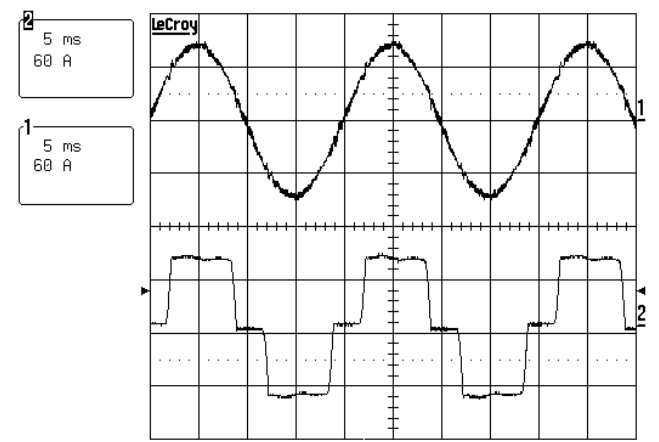

Fig. 23. Steady-state operation compensating up to $25^{\text {th }}$ harmonic for PI-RES controller. Trace 1: $i_{S a}$ (A). Trace 2: $i_{L a}(\mathrm{~A})$.

account in the current controller closed loop transfer function. Doing so, the performed frequency-domain analysis have shown that the PI-RES control is able to compensate up to the $25^{\text {th }}$ harmonic frequency without any delay compensation method. Unlike the PI-RES control, the P-SSI and P-SSISRF compensate up to the $13^{\text {th }}$ harmonic frequency. On the other hand, when the delay compensation is used, all current controllers are stable and able to compensate for the desired harmonics up to the $49^{\text {th }}$ harmonic.

All the resonant current controllers have been implemented for a $25 \mathrm{kVA}$ APF prototype using the dSPACE DS1103 board

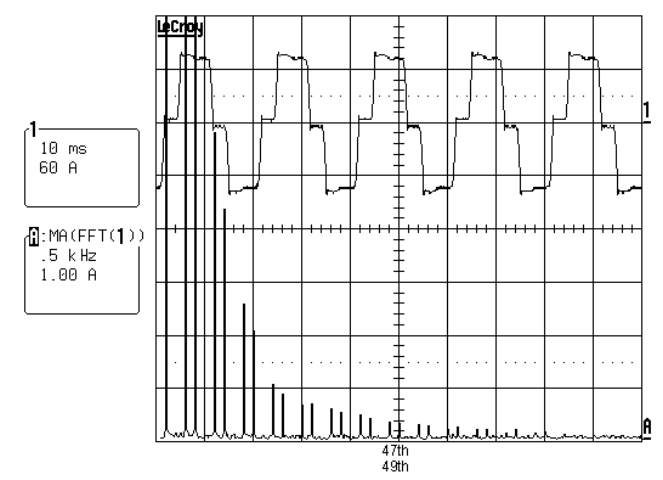

Fig. 24. Inductive load current waveform (phase A) and its Fourier analysis.

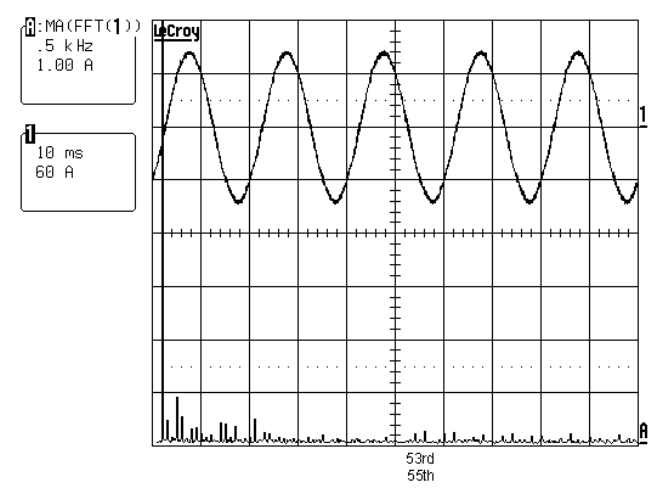

Fig. 25. Fourier analysis for the P-SSI-SRF compensating up to $49^{\text {th }}$ harmonic.

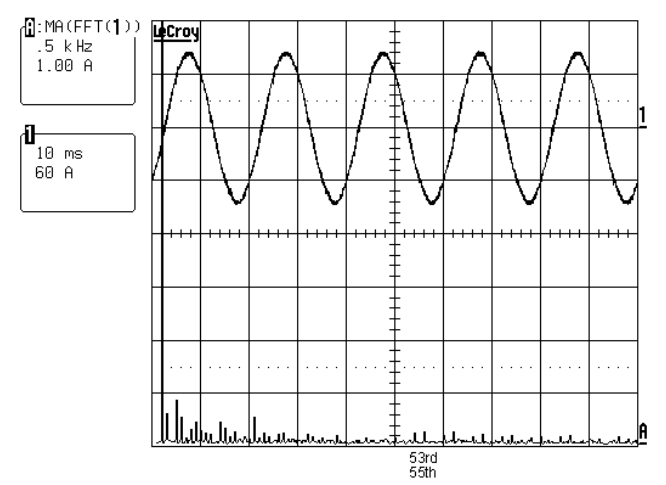

Fig. 26. Fourier analysis for the PI-RES compensating up to $49^{\text {th }}$ harmonic.

to demonstrate the stability limits and the effectiveness of the delay compensation. In industrial applications, the number of compensated harmonics is often limited by the DSP controller computation capability as shown in [13].

\section{REFERENCES}

[1] S. Battacharya, D. M. Divan, and B. Bannerjee. Active filter solutions for utility interface. In Conf. Rec. IEEE ISIE, volume 1, pages 53-63, 1995.

[2] S. Bhattacharya, T. M. Frank, D.M. Divan, and B. Banerjee. Active filter system implementation. IEEE Ind. Applicat. Mag., 4(5):47-63, Sept 1998.

[3] H. Akagi. Trends in active power line conditioners. Conf. Rec. IEEE PESC Power Electron., 9(3):263-268, May 1994.

[4] F. Z. Peng, H. Akagi, and A. Nabae. A novel harmonic power filter. In Conf. Rec. IEEE PESC, volume 2, pages 1151-1159, 1988.

[5] H. Fujita and H Akagi. A practical approach to harmonic compensation in power systems-series connection of passive and active filters. IEEE Trans. Ind. Appl., 27(6):1020-1025, 1991.

[6] S. Srianthumrong and H. Akagi. A mediumvoltage transformerless ac/dc power conversion system consisting of a diode rectifier and a shunt hybrid filter. IEEE Trans. Ind. Appl., 39(3):874-882, 2003.

[7] H. Akagi, Y. Kanagawa, and A. Nabae. Instantaneous 
reactive power compensators comprising switching devices without energy storage components. IEEE Trans. Ind. Appl., 20:625-630, 1984.

[8] R. S. Herrera, P. Salmeron, and H. Kim. Instantaneous reactive power theory applied to active power filter compensation: Different approaches, assessment, and experimental results. IEEE Trans. Ind. Electron., 55(1):184-196, Jan. 2008.

[9] M. El-Habrouk, M. K. Darwish, and P. Metha. Active power filters: A review. In IEE Proc. Elect. Power Appl., volume 147, pages 7-12, 2000.

[10] S. Buso, L. Malesani, and P. Mattavelli. Comparison of current control techniques for active filter applications. IEEE Trans. Ind. Electron., 45(5):722-729, Oct. 1998.

[11] J. Rodríguez, J. Pontt, C. A. Silva, P. Correa, P. Lezana, P. Cortes, and U. Ammann. Predictive current control of a voltage source inverter. IEEE Trans. Ind. Electron., 54(1):495-503, Feb. 2007.

[12] M. P. Kazmierkowski and L. Malesani. Current control techniques for three-phase voltage-source pwm converters: A survey. IEEE Trans. Ind. Electron., 45(5):691-703, Oct. 1998.

[13] L. R. Limongi, R. Bojoi, G. Griva, and A. Tenconi. Digital current-control schemes. IEEE Ind. Electron. Mag., 3(1):20-31, 2009.

[14] M. Sonnenschein and M. Weinhold. Comparison of timedomain and frequency-domain control schemes for shunt active filters. In Conf. Rec. ETEP, volume 9, pages 5-16, 1999.

[15] Y. Sato, T. Ishizuka, K. Nezu, and T. Kataoka. A new control strategy for voltage-type pwm rectifiers to realize zero steady-state control error in input current. IEEE Trans. Ind. Appl., 34(3):480-486, 1998.

[16] D. N. Zmood, D. G. Holmes, and G. Bode. Frequency domain analysis of three phase linear current regulators. IEEE Trans. Ind. Appl., 37(2):601-610, March 2001.

[17] D. N. Zmood and D. G. Holmes. Stationary frame current regulation of pwm inverters with zero steady-state error. IEEE Trans. Power Electron., 18(3):814-822, May 2003.

[18] X. Yuan, W. Merk, H. Stemmler, and J. Allmeling. Stationary-frame generalized integrators for current control of active power filters with zero steady-state error for current harmonics of concern under unbalanced and distorted operating conditions. IEEE Trans. Ind. Appl., 38(2):523-532, March 2002.

[19] S. Fukuda and R. Imamura. Application of a sinusoidal internal model to current control of three-phase utilityinterface converters. IEEE Trans. Ind. Electron., 52(2):420-426, April 2005.

[20] R. Grinó, R. Cardoner, R. Costa-Castelló, and E. Fossas. Digital repetitive control of a three-phase four-wire shunt active filter. IEEE Trans. Ind. Electron., 54(3):14951503, June 2007.

[21] P. Mattavelli and F. P. Marafao. Repetitive-based control for selective harmonic compensation in active power filters. IEEE Trans. Ind. Electron., 51(5):1018-1024, 2004.

[22] R. Bojoi, G. Griva, V. Bostan, M. Guerriero, F. Farina, and F. Profumo. Current control strategy for power conditioners using sinusoidal signal integrators in synchronous reference frame. IEEE Trans. Power Electron., 20(6):1402-1412, Nov. 2005.

[23] L. Asiminoaei, C. Lascu, F. Blaabjerg, and I. Boldea. New current control structure for shunt active power filters. In Conf. Rec. IEEE IAS, pages 183-190, 2006.

[24] C. Lascu, L. Asiminoaei, I. Boldea, and F. Blaabjerg. High performance current controller for selective harmonic compensation in active power filters. IEEE Trans. Power Electron., 22(5):1826-1835, 2007.

[25] C. Lascu, L. Asiminoaei, I. Boldea, and F. Blaabjerg. Frequency response analysis of current controllers for selective harmonic compensation in active power filters. IEEE Trans. Ind. Electron., 56(2):337-347, Feb. 2009.

[26] F. Briz, M. W. Degner, and R. D. Lorenz. Analysis and design of current regulators using complex vectors. IEEE Trans. Ind. Appl., 36(3):817-825, May 2000.

[27] D. Basic, V. S. Ramsden, and P. K. Muttik. Harmonic filtering of high-power 12-pulse rectifier loads with a selective hybrid filter system. IEEE Trans. Ind. Electron., 48(6):1118-1127, Dec. 2001.

[28] K. Ogata. Modern Control Engineering. Prentice Hall, $3^{\text {rd }}$ Edition, 1997.

\section{BIOGRAPHIES}

Leonardo Rodrigues Limongi was born in Recife, Brazil, in 1978. He received the M.Sc. degree in electrical engineering from the Universidade Federal de Pernambuco, Recife, Brazil, in 2006, and the Ph.D. degree from the Politecnico di Torino, Turin, Italy, in 2009. Since 2010, he has been at the Electrical Engineering Department, Universidade Federal de Pernambuco, where he is currently a Professor of Electrical Engineering. He is the author of more than 20 papers published in international conference proceedings and technical journals. His research interests include the fields of power electronics dedicated to power conditioning systems and distributed generation.

Daniel Roiu was born in Iasi, Romania, in 1981. He received the M.Sc. degree in electrical engineering from the Technical University Gh. Asachi Iasi, Iasa, Romania, in 2005, and a joint Ph.D. degree from the Technical University Gh. Asachi Iasi and the Politecnico di Torino, Turin, Italy, in 2009. $\mathrm{He}$ is currently a Researcher in FIAT Research Centre, Turin. His scientific interests include power electronics devices for power quality improvement and distributed generation interfaces.

Radu Bojoi received the M.Sc. degree in electrical engineering from the Technical University Gh. Asachi Iasi, Iasi, Romania, in 1993, and the Ph.D. degree from the Politecnico di Torino, Turin, Italy, in 2003. From 1994 to 1999, he was an Assistant Professor in the Department of Electrical Drives and Industrial Automation, Technical University of Iasi. In 2004, he joined the Electrical Engineering Department, Politecnico di Torino, where he is currently an Associate Professor. He is the author of more than 60 papers published in international conference proceedings and technical journals. His scientific interests 
include the design and development of DSP- and FPGA-based advanced control systems in the fields of power electronics, high performance electrical drives, and power-conditioning systems. Dr. Bojoi received the IPEC First Prize Paper Award in 2005 .

Alberto Tenconi received the M.Sc. and the Ph.D. degrees in electrical engineering from the Politecnico di Torino, Turin, Italy, in 1986 and 1990, respectively. From 1988 to 1993, he was with the Electronic System Division of the FIAT Research Center, where he was engaged in the development of electrical vehicle drive systems. He then joined the Department of Electrical Engineering, Politecnico di Torino, where he is currently a Full Professor. He is the author of more than 120 papers published in international journals and conference proceedings. $\mathrm{He}$ is a reviewer of international journals and Associate Editor of IEEE Transactions on Industrial Electronics. His fields of interest are high-performance drive design, new power electronic device applications, and nonconventional electric machines development. He has participated both as a designer and as a scientist in many national and European research programs. 